\title{
A homozygous mutation in HESX1 is associated with evolving hypopituitarism due to impaired repressor-corepressor interaction
}

\author{
Luciani R. Carvalho, ${ }^{1}$ Kathryn S. Woods, ${ }^{2}$ Berenice B. Mendonca, ${ }^{1}$ Nathalie Marcal, ${ }^{3}$ \\ Andrea L. Zamparini, ${ }^{4}$ Stefano Stifani, ${ }^{3}$ Joshua M. Brickman, ${ }^{4}$ Ivo J.P. Arnhold, ${ }^{1}$ \\ and Mehul T. Dattani ${ }^{2}$ \\ ${ }^{1}$ Unidade de Endocrinologia do Desenvolvimento, Laboratório de Hormônios e Genética Molecular, \\ Laboratorio de Investigação Medica/42, Disciplina de Endocrinologia, Hospital das Clinicas de \\ Faculdade de Medicina da Universidade de São Paulo, São Paulo, Brazil \\ ${ }^{2}$ London Centre for Paediatric Endocrinology, Biochemistry, Endocrinology and Metabolism Unit, \\ Institute of Child Health and Great Ormond Street Children's Hospital, London, United Kingdom \\ ${ }^{3}$ Montreal Neurological Institute, McGill University, Montreal, Quebec, Canada \\ ${ }^{4}$ Institute for Stem Cell Research, Edinburgh, United Kingdom
}

\begin{abstract}
The paired-like homeobox gene expressed in embryonic stem cells Hesx1/HESX1 encodes a developmental repressor and is expressed in early development in a region fated to form the forebrain, with subsequent localization to Rathke's pouch, the primordium of the anterior pituitary gland. Mutations within the gene have been associated with septo-optic dysplasia, a constellation of phenotypes including eye, forebrain, and pituitary abnormalities, or milder degrees of hypopituitarism. We identified a novel homozygous nonconservative missense mutation (I26T) in the critical Engrailed homology repressor domain (eh1) of HESX1, the first, to our knowledge, to be described in humans, in a girl with evolving combined pituitary hormone deficiency born to consanguineous parents. Neuroimaging revealed a thin pituitary stalk with anterior pituitary hypoplasia and an ectopic posterior pituitary, but no midline or optic nerve abnormalities. This I26T mutation did not affect the DNAbinding ability of HESX1 but led to an impaired ability to recruit the mammalian Groucho homolog/Transducin-like enhancer of split-1 (Gro/TLE1), a crucial corepressor for HESX1, thereby leading to partial loss of repression. Thus, the novel pituitary phenotype highlighted here appears to be a specific consequence of the inability of HESX1 to recruit Groucho-related corepressors, suggesting that other molecular mechanisms govern HESX1 function in the forebrain.
\end{abstract}

J. Clin. Invest. 112:1192-1201 (2003). doi:10.1172/JCI200318589.

\section{Introduction}

The pituitary gland is a midline structure, consisting of three lobes: the anterior and posterior lobes and a smaller intervening intermediate lobe. The posterior

Received for publication April 8, 2003, and accepted in revised form August 5, 2003.

Address correspondence to: Mehul T. Dattani, London Centre for Paediatric Endocrinology, Biochemistry, Endocrinology and Metabolism Unit, Institute of Child Health and Great Ormond Street Children's Hospital, 30 Guilford Street, London

WC1N 1EH, United Kingdom. Phone: 44-20-7905-2657; Fax: 44-20-7404-6191; E-mail: mdattani@ich.ucl.ac.uk.

Luciani R. Carvalho and Kathryn S. Woods contributed equally to this work.

Conflict of interest: The authors have declared that no conflict of interest exists.

Nonstandard abbreviations used: homeobox gene expressed in embryonic stem cells (Hesx1); septo-optic dysplasia (SOD); growth hormone (GH); isolated GH deficiency (IGHD); Paired ( $p r d)$; pituitary-specific transcription factor 1 (Pit1); prophet of Pit1 (PROP1); engrailed homology domain (eh1); Transducin-like enhancer of split (TLE); Groucho homolog/Transducin-like enhancer of split-1 (Gro/TLE1); days post coitum (dpc); prd-like homeodomain transcription factor 1 (Pitx1); combined pituitary hormone deficiency (CPHD); posterior pituitary lobe (PPL); GH-releasing hormone (GHRH); standard deviation score (SDS); thyroid stimulating hormone (TSH). lobe arises from neural ectoderm, whereas the anterior and intermediate lobes originate from oral ectodermal tissue $(1,2)$. The gland is a central regulator of growth, homeostasis, and reproduction, and these complex functions are mediated by six different hormones secreted by five different cell types that are present within the mature anterior pituitary gland. Fate map analyses in the mouse have shown that the most anterior part of the neural plate, the anterior neural ridge (ANR), will later give rise to non-neural structures such as the anterior pituitary, the nasal cavity ectoderm, and the olfactory placode, while the anterior neural structures, namely the hypothalamus, posterior pituitary, optic vesicles, and ventral forebrain, derive from the neural plate tissue immediately adjacent to the ANR.

The homeobox gene expressed in embryonic stem cells Hes $x 1$ regulates some of the earliest stages in pituitary development. Mice homozygous for a mutation in Hesx 1 display multiple defects in placodally derived anterior structures including the eye, olfactory epithelium, forebrain, and pituitary $(3,4)$. A similar constellation of phenotypes is associated with the human syndrome septooptic dysplasia (SOD), a familial form of which has been associated with a homozygous missense mutation 
(R160C) in the human homolog HESX1 (3). A further three heterozygous mutations (S170L, T181A, and Q6H) have been described in association with milder pituitary phenotypes characterized by isolated growth hormone deficiency (IGHD) with or without an ectopic posterior pituitary $(5,6)$. More recently, a de novo heterozygous mutation (306/307insAG) within exon 2 was associated with panhypopituitarism and anterior pituitary hypoplasia, an ectopic posterior pituitary, and left optic nerve hypoplasia in a Japanese patient (7).

HESX1 is a member of the Paired (prd) class of homeodomain proteins and is a transcriptional repressor. $P r d-$ class homeodomains can cooperatively dimerize on palindromic binding sites containing two core TAAT homeodomain sites. Heterodimerization on dimeric sites could result in preferential interactions between activator and repressor homeoproteins, leading to heteromeric repression. Hesx 1 and a second prd-like homeodomain factor, prophet of pituitary-specific transcription factor 1 (prophet of Pit1, called Prop1), are expressed in spatially and temporally distinct and overlapping domains during pituitary development. Analysis of the Ames dwarf (Prop $\left.1^{d f}\right)$ mouse, which bears a hypomorphic mutation in the homeobox of Prop1, and of human patients with PROP1 mutations (8) has suggested that Prop1 is required for ventral proliferation and determination of four anterior cell lineages: somatotropes, lactotropes, thyrotropes, and gonadotropes. Additionally, variable cortisol deficiency is observed in a number of human patients with a PROP1 mutation (9), although the underlying mechanism appears unclear. Attenuation of Hesx 1 expression in the developing murine pituitary coincides with the Prop1-dependent progression of cellular differentiation, and this suggests that the temporal regulation of Hesx 1 is crucial for the cellular differentiation activated by Prop $1(10,11)$. Prop 1 and Hesx 1 can both bind a palindromic site; PrdQ (three copies of the PrdQ element [GATCCGACTAATGGAATTA]) and both Prop1 and Hesx1 homodimers or a Prop1/Hesx1 heterodimer can recognize this sequence (12).

Hesx 1 is a promoter-specific repressor that can suppress the activity of activator proteins bound within the same DNA recognition element in a process known as heteromeric or cooperative repression (6). Consequently, Hesx 1 may act in the context of a Hesx $1 /$ Prop 1 complex to suppress the activity of Prop1 (13). Heteromeric repression of homeodomain-containing activators by Hesx 1 may require additional factors. Potential cofactors identified to date include the nuclear receptor corepressor $(\mathrm{N}-\mathrm{CoR}), \mathrm{mSin} 3 \mathrm{~A} / \mathrm{B}$, and histone deacetylases (e.g., histone deacetylase complex 2, HDAC2) (14). Additionally, the 185-AA open reading frame contains within its $\mathrm{N}$-terminus a highly conserved domain (engrailed homology domain, eh1) characterized by 7 AA residues (AAs 21-27) that is implicated in transcriptional repression $(6,13)$. This sequence of AAs is contained within a minimal repression domain defined as both necessary and sufficient for repression by a GAL4-Hesx1 fusion in transient transfections (6). In the Drosophila melanogaster proteins
Goosecoid and Engrailed, the eh1 sequence is necessary for recruitment of the corepressor Groucho $(15,16)$. In Goosecoid, eh1 also mediates heteromeric repression (17). Murine Hesx1 has recently been shown to interact with Transducin-like enhancer of split-1 (Gro/TLE1), the mammalian homolog of the $D$. melanogaster corepressor Groucho, and this interaction is dependent on eh1 (13). Gro/TLE1 is coexpressed with Hesx 1 within Rathke's pouch between 9 days post coitum $(\mathrm{dpc})$ and $13.5 \mathrm{dpc}$, after which expression is switched off (13). The Gro/TLE1-dependent repression by Hesx 1 appears to be crucial to the role of Hesx 1 in regulating the proliferation patterns of pituitary progenitor lineages. Transgenic mice in which ectopic Hesx1 and Gro/TLE1 are expressed from the prd-like homeodomain transcription factor 1 (Pitx1) promoter (to maintain expression throughout pituitary development) phenocopy the effects of Prop1 gene mutations, with a complete absence of Pit1-dependent cell lineages and gonadotropes (13).

To date, a broad spectrum of phenotypes such as SOD, combined pituitary hormone deficiency (CPHD), and IGHD with an undescended/ectopic posterior pituitary lobe (PPL) have been observed in patients with HESX1 mutations (3, 5-7). Some of these mutations have been associated with effects on the binding of HESX1 to DNA. However, none of these mutations has as yet been linked specifically to the repressor activity of HESX1. To attempt to link specific phenotypes to aspects of HESX1 function, we screened a cohort of Brazilian patients with IGHD and CPHD with an ectopic, nonvisualized, or eutopic PPL. We identified a novel homozygous mutation within eh1 in HESX1 in a child with evolving CPHD, which to our knowledge is the first to be described within this highly conserved region that is present in a number of developmental repressors. We have for the first time, to our knowledge, established that this mutation impairs the function of HESX1 as a transcriptional repressor, and that this reduction in transcriptional repression is mediated by impaired interaction with Gro/TLE corepressor proteins.

\section{Methods}

Patient recruitment. Informed parental consent, patient assent, and approval by the Hospital Ethics Committee were obtained before initiation of the studies. Sixty-five Brazilian patients with IGHD or CPHD without CNS malformation and either an ectopic PPL $(n=43)$, a nonvisualized PPL $(n=12)$, or a eutopic PPL $(n=10)$ were studied at the Hospital das Clinicas de Faculdade de Medicina da Universidade de São Paolo.

Growth hormone-releasing hormone test. Intravenous growth hormone-releasing hormone (GHRH; Ferring Arzneimittel GmbH, Kiel, Germany) was administered, 4 years after the withdrawal of growth hormone $(\mathrm{GH})$ treatment, in increasing doses over a 3-day period: 0.1 $\mu \mathrm{g} / \mathrm{kg}$ on day $1,0.33 \mu \mathrm{g} / \mathrm{kg}$ on day 2 , and $1.0 \mu \mathrm{g} / \mathrm{kg}$ on day 3 . The increasing doses were used in an attempt to prime the anterior pituitary. GH concentrations were measured at $-15,0,5,15,30,45,60,90$, and 120 minutes 
with respect to the administration of GHRH. A combined pituitary stimulation test was performed as previously described (18).

$P C R$ and automated sequencing analysis of DNA. The four exons encoding the open reading frame of the HESX1 gene were amplified by PCR using intronic primers as previously described (3). The following PCR conditions were used: $94^{\circ} \mathrm{C}$ for 5 minutes, followed by 30 cycles of $94^{\circ} \mathrm{C}$ for 30 seconds, $55^{\circ} \mathrm{C}$ for 30 seconds, and $72^{\circ} \mathrm{C}$ for 30 seconds, with a final extension of $72^{\circ} \mathrm{C}$ for 10 minutes. PCR products were treated with shrimp alkaline phosphatase and exonuclease I (PCR product presequencing kit; Amersham Life Sciences Inc., Cleveland, Ohio, USA) prior to sequencing using the ABI PRISM BigDye Terminator kit (Perkin-Elmer Applied Biosystems, Foster City, California, USA). The products were then directly sequenced using an ABI PRISM 310 Genetic Analyzer automatic DNA sequencer (PerkinElmer Applied Biosystems).

Bsu36I restriction assays. The $\mathrm{T} 77 \mathrm{C}$ mutation introduces a restriction site for $B s u 36 \mathrm{I}$ in exon 1 . Exon 1 was therefore amplified by PCR, and $5 \mu \mathrm{l}$ of the PCR product was digested with $0.5 \mu \mathrm{l}$ Bsu36I enzyme $(10 \mathrm{U} / \mu \mathrm{l})$. The enzyme cleaves the 219-bp fragment from exon 1 into two fragments of similar size (105 and $114 \mathrm{bp}$ ) in patients homozygous for I26T. In the wild-type allele, only the undigested 219-bp fragment could be detected. Allele-tracking studies. Haplotype analysis was performed using three markers flanking HESX1 within a 1.00-Mb range (D3S3616, D3S3532, and D3S1295). Primers were $5^{\prime}$ fluorescently labeled with Fam or Hex (Invitrogen Corp., San Diego, California, USA). In brief, $10 \mathrm{ng}$ of genomic DNA was used in a PCR with 20 pmol of each primer, $1.5 \mathrm{mM} \mathrm{MgCl}_{2}, 2.5 \mathrm{U}$ Taq gold polymerase (AmpliTaq Gold; Applied Biosystems, Foster City, California, USA), and $100 \mu \mathrm{M}$ dNTPs in a 50$\mu l$ reaction. PCRs were performed in a Mastercycler Gradient (Eppendorf AG, Hamburg, Germany). Resultant PCR products were analyzed on the ABI PRISM 310 Genetic Analyzer (Perkin-Elmer Applied Biosystems), and results were evaluated using GeneScan (Applied Biosystems). Haplotype segregation analysis was performed in two pedigrees.

Purification of recombinant proteins and gel shift assays. HESX1 proteins were expressed using the TNT Quick Coupled Transcription/Translation System (Promega Corp., Madison, Wisconsin, USA). Briefly, HESX1 cDNA [HESX1, HESX1(I26T), and HESX1(50-185)] was subcloned downstream of the T7 promoter into the expression vector PET30a. Standard rabbit-reticulocyte systems commonly use RNA synthesized in vitro from SP6, T3, or T7 RNA polymerase. The TNT Quick Coupled Transcription/Translation System simplifies the process by combining the RNA polymerase, nucleotides, salts, and Recombinant RNasin Ribonuclease Inhibitor with the reticulocyte lysate to form a single TNT Quick Master Mix (Promega Corp.).

Electrophoretic mobility shift assays were performed as previously described $(6,19)$ using the P3 probe (5' AGCTTGAGTCTAATTGAATTACTGTAC $3^{\prime}$ ) and increasing amounts of in vitro-translated protein $(1 \mu \mathrm{l}, 5 \mu \mathrm{l}, 10 \mu \mathrm{l}$, and $20 \mu \mathrm{l})$.

All HESX1 proteins were also expressed and purified using the pET system (Novagen, Madison, Wisconsin, USA). Briefly, HESX1 cDNA [HESX1, HESX1(I26T), and HESX1(50-185)] was subcloned into PET30a, an expression vector containing six histidine residues upstream of the multiple cloning site. Following induction by isopropyl- $\beta$-D-thiogalactopyranoside (IPTG), insoluble cell extracts from the Escherichia coli strain BL21(DE3) were solubilized in $20 \mathrm{mM}$ Tris ( $\mathrm{pH} 8.0$ ), $100 \mathrm{mM} \mathrm{KCl}$, $10 \%$ (wt/vol) glycerol, $8 \mathrm{M}$ urea, $1 \mathrm{mM}$ PMSF, and 30 $\mathrm{mM}$ imidazole. This solute was fractionated by nickel affinity $\left(\mathrm{Ni}^{2+}\right)$ chromatography. HESX1-containing fractions were eluted using $250 \mathrm{mM}$ imidazole. The protein was renatured by dialysis against $100 \mathrm{mM}$ Tris ( $\mathrm{pH} 7.5$ ), $600 \mathrm{mM} \mathrm{KCl}, 10 \mathrm{mM} \mathrm{MgCl} 2,20 \%$ (wt/vol) glycerol, $0.1 \%$ Nonidet P40, $2 \mathrm{mM}$ DTT, and $1 \mathrm{mM}$ PMSF containing decreasing amounts of urea. Recombinant protein could then be quantified using the Bradford protein assay (Bio-Rad Laboratories Ltd., Hemel Hempstead, Hertfordshire, United Kingdom). Plasmid constructs. Gal4-HESX1 fusion proteins [Gal4HESX1, Gal4-HESX1(I26T), and Gal4-HESX1(50-185)] were constructed as previously described $(6,20)$. The reporter construct containing reiterated 17-mers upstream of the SV40 promoter has been described previously (6); the (P3)E4 reporter was a gift from M. Tada, as was the pcDNA3 Bix plasmid. The Gro/TLE1 fusion plasmid, containing the activation domain of the herpes simplex transactivator VP16 upstream of Gro/TLE1, was described previously (21). Constructs pCMV2FLAG-HESX1 and PCMV2-FLAG-HESX1(I26T) were generated by subcloning of the appropriate BglII/SalI restriction fragments into the BglII and SalI sites of pCMV2-FLAG. Plasmid PCMV2-FLAG-Hes1 $\Delta$ WRPW has been described previously (22).

Cell culture and transfections. Both $\mathrm{CHO}$ and Cos-7 cells were cultured in DMEM supplemented with $10 \%$ FBS and $2 \mathrm{mM}$-glutamine. Transient transfections were carried out using LipofectAMINE (Invitrogen Corp.) following the manufacturer's protocol with modifications. Briefly, $1 \times 10^{5}$ cells were seeded into each well on a 12 -well plate 24 hours before transfection. Cells were transfected with 75 ng of pRL-SV40 Renilla luciferase vector (Promega Corp.) to control for transfection efficiency and $100 \mathrm{ng}$ of the appropriate firefly luciferase reporter. The DNA concentration of the other transfected plasmids varied depending on experimental protocol as indicated in the figure legends, but the total amount of DNA transfected per well was normalized to $1.2 \mu \mathrm{g}$ by addition of the appropriate amount of empty expression vector. Cells were incubated with transfection media (DNA plus OptiMEM; Invitrogen Corp.) for approximately 15 hours and then re-fed with normal growth media. Cells were collected 24 hours later and assayed for luciferase activity following the DualLuciferase protocol (Promega Corp.). 
Coimmunoprecipitation studies. Human HEK 293 cells were transfected and whole-cell lysates were prepared and subjected to immunoprecipitation as described previously (23), followed by Western blotting with antibodies against either the FLAG epitope (Sigma Aldrich, St. Louis, Missouri, USA) or Gro/TLE proteins (pan-TLE antibodies) (24).

\section{Results}

Phenotype. A total of 65 patients with IGHD or CPHD without apparent CNS malformation were screened for mutations in HESX1. A novel homozygous T77C transition within exon 1 , resulting in the nonconservative substitution of isoleucine by threonine at codon 26 (I26T), was identified in a single patient with CPHD. The position of this mutation relative to the HESX1 coding sequence is illustrated in Figure $1 \mathrm{~b}$.

The patient first presented at the age of 5 years with short stature (height $90 \mathrm{~cm} ;-4.6$ standard deviation score $[$ SDS]), although poor growth was noticed in the first year of life. Her bone age, as estimated by the Greulich-Pyle method, was delayed by 3 years. Her birth weight was $3.0 \mathrm{~kg}$, and her length at birth was $43 \mathrm{~cm}$. Her parents were first-degree cousins and of normal height, the father $166 \mathrm{~cm}(-0.7 \mathrm{SDS})$ and the mother $159 \mathrm{~cm}(-0.4$ SDS $)$. Her adult sister achieved a final height of $160 \mathrm{~cm}(-0.4 \mathrm{SDS})$, and that of her adult brother is $170 \mathrm{~cm}(-0.7 \mathrm{SDS})$.

The diagnosis of GH deficiency was established using two distinct tests for $\mathrm{GH}$ secretion: clonidine (basal GH $<0.25 \mathrm{ng} / \mathrm{ml}$, peak $\mathrm{GH}<0.25 \mathrm{ng} / \mathrm{ml}$ ) and insulininduced hypoglycemia (Table 1 ). Recombinant GH treatment was commenced at $0.023 \mathrm{mg} / \mathrm{kg} / \mathrm{d}$ over the first 3 years, and this dose was increased to $0.03 \mathrm{mg} / \mathrm{kg} / \mathrm{d}$ for a further 2 years and $0.05 \mathrm{mg} / \mathrm{kg} / \mathrm{d}$ for the next 4 years. When the patient reached the age of 15 years, no signs of puberty were detectable. A gonadotropin-releasing hormone stimulation test confirmed gonadotropin deficiency (Table 1), and pubertal induction was commenced using conjugated estrogen, with the later addition of medroxyprogesterone acetate. At the age of 19 , her final height was $165 \mathrm{~cm}(+0.5 \mathrm{SDS})$, well above her target height of $152 \mathrm{~cm}$, and she weighed $65 \mathrm{~kg}(+1.1$ SDS). Fundoscopy revealed normal optic discs, with no evidence of optic nerve hypoplasia. At the age of 21 years, thyroxin concentrations were slightly reduced (free thyroxin $0.4 \mathrm{ng} / \mathrm{dl}$, normal $0.6-1.54 \mathrm{ng} / \mathrm{dl}$; total thyroxin 4.9 $\mu \mathrm{g} / \mathrm{dl}$, normal $4.5-12.0 \mu \mathrm{g} / \mathrm{dl}$ ), and re-evaluation of the hypothalamo-pituitary axis confirmed $\mathrm{GH}$ and gonadotropin deficiencies with evolving cortisol and thyroid stimulating hormone (TSH) deficiencies (Table $1)$. The low thyroxin concentration coupled with a

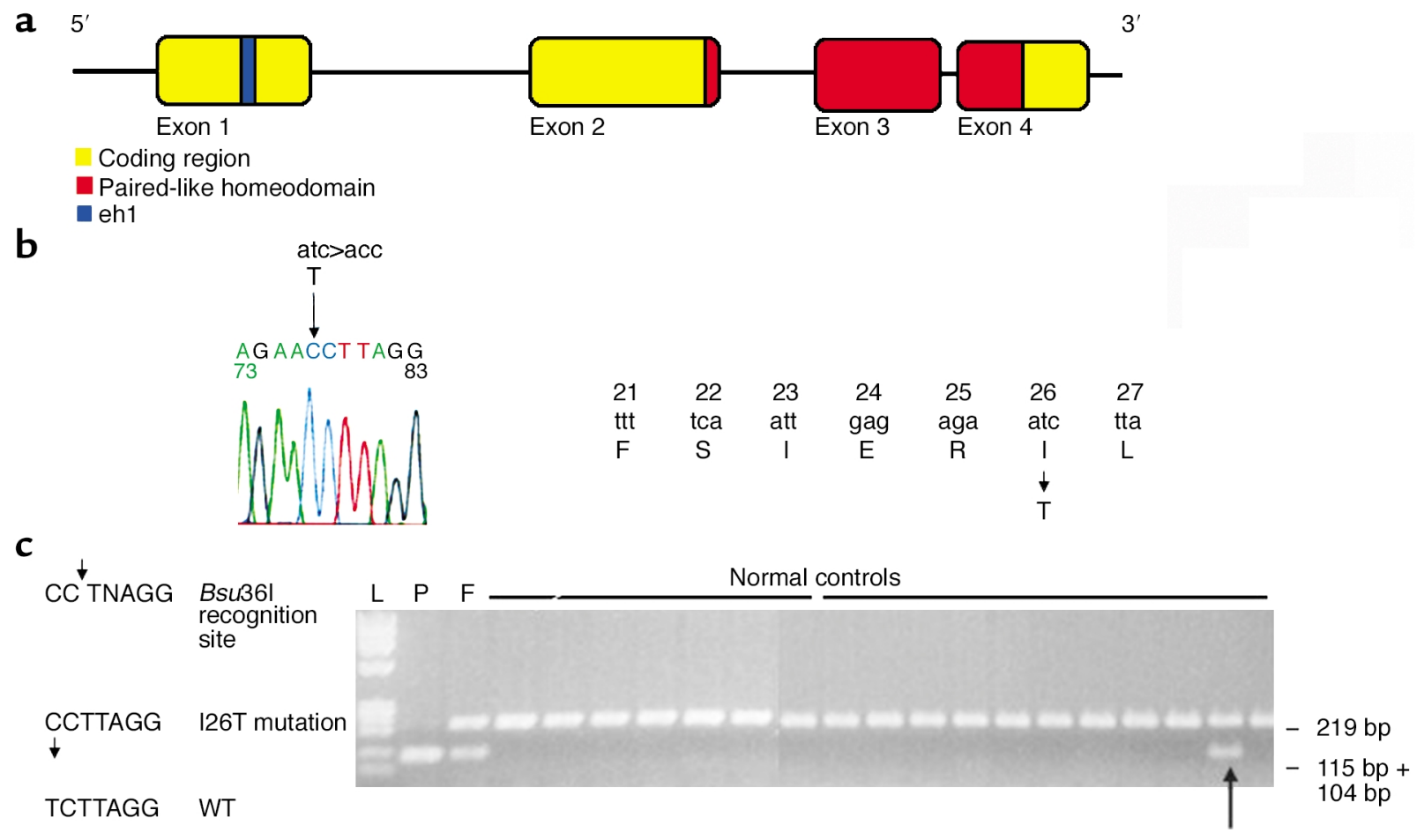

\section{Figure 1}

Schematic of the HESX1 gene, HESX1(I26T) mutation, and Bsu36I restriction digest assay within a normal Brazilian population. (a) Genomic and protein structure of HESX1. HESX1 consists of four exons encoding a 185-AA protein. The engrailed homology domain (AAs 21-27) is encoded by exon 1 and the prd-like homeodomain by exons 2-4. (b) Homozygous missense mutation within exon 1 leads to the substitution of an isoleucine residue (codon 26) by threonine in the engrailed homology domain eh1. (c) Bsu36l restriction digests in pedigree 1 (F, father; P, patient) and 18 control samples. Digestion of a homozygous sample results in one band (104 + 115 bp fragment size), digestion of a heterozygous sample results in two bands (a 219-bp band from the normal allele and a band with the 104-bp and 115-bp fragments from the mutant allele), and digestion of a homozygous wild-type sample results in a single 219-bp band. The arrow indicates a normal control who is heterozygous for HESX1(I26T). 
Table 1

Clinical phenotype of patient with I26T substitution of HESX1

\begin{tabular}{|c|c|c|c|c|c|c|c|c|c|c|c|c|c|c|}
\hline \multirow[t]{2}{*}{$\begin{array}{l}\text { Age } \\
(y r)\end{array}$} & \multirow[t]{2}{*}{$\begin{array}{c}\text { Nadir blood } \\
\text { glucose }(\mathrm{mg} / \mathrm{dl})\end{array}$} & \multirow{2}{*}{$\begin{array}{c}\mathrm{GH} \\
(\mathrm{ng} / \mathrm{ml}) \\
\mathrm{P}\end{array}$} & \multicolumn{2}{|c|}{$\begin{array}{l}\text { Cortisol } \\
(\mu \mathrm{g} / \mathrm{dl})\end{array}$} & \multicolumn{2}{|c|}{$\begin{array}{c}\mathrm{PRL} \\
(\mathrm{ng} / \mathrm{ml})\end{array}$} & \multicolumn{2}{|c|}{$\begin{array}{l}\text { FSH } \\
(\mathrm{U} / \mathrm{I})\end{array}$} & \multicolumn{2}{|c|}{$\begin{array}{l}\mathrm{LH} \\
(\mathrm{U} / \mathrm{I})\end{array}$} & \multicolumn{2}{|c|}{$\begin{array}{c}\text { TSH } \\
(\mu \mathrm{U} / \mathrm{ml})\end{array}$} & \multirow{2}{*}{$\begin{array}{c}\mathrm{FT4} \\
(\mathrm{ng} / \mathrm{dl}) \\
\mathrm{B}\end{array}$} & \multirow{2}{*}{$\begin{array}{c}\mathrm{T} 4 \\
(\mu \mathrm{g} / \mathrm{dl}) \\
\mathrm{B}\end{array}$} \\
\hline & & & B & $\mathrm{P}$ & B & $\mathrm{P}$ & $B$ & $\mathrm{P}$ & B & $\mathrm{P}$ & B & P & & \\
\hline 5 & 19 & $<0.25$ & $15^{\mathrm{A}}$ & 27 & $7^{A}$ & 14 & $3^{\mathrm{A}}$ & 9 & $4^{\mathrm{A}}$ & 8 & 1.7 & 8.4 & - & 8 \\
\hline 15 & & & - & - & & & $<1^{\mathrm{B}}$ & $<1$ & $<0.6^{B}$ & $<0.6$ & & & 1.0 & 6.0 \\
\hline 21 & 19 & $<0.1$ & $3^{\mathrm{B}}$ & 5 & $6^{\mathrm{B}}$ & 13 & $<1^{\mathrm{B}}$ & $<1$ & $<0.6^{\mathrm{B}}$ & $<0.6$ & 0.7 & 5.6 & 0.4 & 4.9 \\
\hline
\end{tabular}

Basal (B) and peak (P) hormone levels after intravenous administration of insulin ( $0.1 \mathrm{U} / \mathrm{kg}$ body weight) plus TRH (200 $\mu \mathrm{g})$ plus gonadotropin-releasing hormone ( $100 \mu \mathrm{g})$. Evolving pituitary hormone deficiency is demonstrated by early $\mathrm{GH}$ deficiency followed by the diagnosis of hypogonadotropic hypogonadism

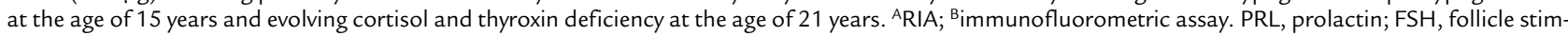
ulating hormone; LH, luteinizing hormone; FT4, free thyroxin; T4, total thyroxin.

normal TSH response to the combined pituitary test is commonly observed in hypothalamo-pituitary disease $(25,26)$ and cannot differentiate between hypothalamic and pituitary disease. To assess whether the GH deficiency was hypothalamic in origin, the patient was reevaluated after withdrawal of GH treatment using GHRH at the age of 22.3 years. GH concentrations were undetectable at all time points over the 3 -day period.

The neuroanatomy associated with the phenotype is shown in Figure 2. Neuroimaging of the hypothalamopituitary region using MRI at 11 and 21 years of age revealed a thin but continuous pituitary stalk (measuring $1.7 \mathrm{~mm}$ proximally and distally at age 11 years; Figure 2a) (27), reduced anterior pituitary height reflecting anterior pituitary hypoplasia (measuring $3.8 \mathrm{~mm}$ at 21 years; $-7.7 \mathrm{SD}$ according to Argyropoulou et al. [ref. 28]; Figure 2 , $a$ and $b$ ), and an ectopic PPL (data not shown). The MRI scan also showed that the patient has normal optic nerves and no forebrain defect (Figure 2, a and b, compared with a normal subject, $\mathrm{c}$ and $\mathrm{d}$ ).

Genetic analysis. Rapid detection of the T77C/I26T allele of HESX1 was made possible by the PCR-based assay shown in Figure 1c. The T77C substitution within exon 1 in the HESX1 coding region creates a new $B s u 36$ I restriction site, which facilitated the screening of a Brazilian control cohort for this mutation. Of the 100 normal individuals screened, one was shown to be heterozygous for the I26T mutation, representing a $0.5 \%$ incidence of the mutant allele within this Brazilian population (Figure 1c). Since the index patient with the homozygous I26T mutation was not related to the phenotypically normal control individual who was heterozygous for this mutation, we investigated the possibility of a common ancestral origin of the mutant allele, using highly polymorphic HESX1-linked markers. Figure 3 shows linkage analysis for the families of both I26T-carrying individuals. In both cases, HESX1(I26T) cosegregated with several closely linked markers (haplotype: 8, 2, HESX1(I26T), 4), whereas wild-type alleles of HESX1 were found segregating with a number of different haplotypes. In pedigree 1 (the family of the index patient), the affected individual IV:1 is homozygous with respect to both HESX1(I26T) and the closely linked markers. The phenotypically normal sister, IV:2, is heterozygous with respect to both HESX1 and the surrounding markers and has inherited the HESX1(I26T) mutant allele from her mother. Figure 3 also shows that in the pedigree of the normal control (pedigree 2, I:1), HESX1(I26T) is also associated with the same set of linked markers (8, 2, HESX1(I26T), 4) as in the family of the index patient. I: 1 and her daughter II: 1 are both heterozygous for HESX1(I26T) and have the same HESX1-linked haplotype, whereas the husband of I:1 (I:2) is wild type with respect to HESX1 and carried two different sets of linked markers. Table 2 shows the frequency of alleles for each of the HESX1linked markers (based on 30 normal Brazilian individuals). Each marker has between six and nine alleles, and
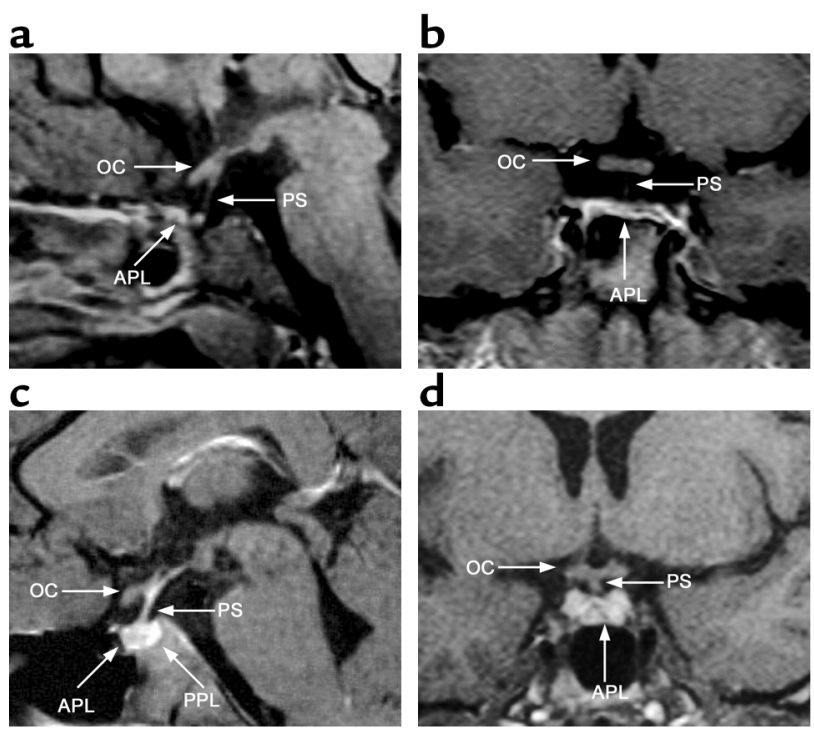

\section{Figure 2}

Neuroimaging studies of the patient with the HESX1(I26T) mutation ( $\mathbf{a}$ and $\mathbf{b}$ ) and a normal subject (c and $\mathbf{d}$ ). (a) Sagittal MRI scan of the patient homozygous for HESX1(I26T) at 21 years, showing a thin, continuous pituitary stalk (PS), a normal optic chiasm (OC), and a severely hypoplastic anterior pituitary lobe (APL). (b) Coronal MRI scan of the patient at 11 years, showing a hypoplastic anterior pituitary lobe and a normal optic chiasm, with a thin pituitary stalk that is not clearly visualized. (c) Normal sagittal MRI scan showing a normal anterior pituitary lobe, a normal pituitary stalk, a normally sited PPL, and a normal optic chiasm. (d) Normal coronal MRI scan showing a normal anterior pituitary lobe and a normal optic chiasm with a normal pituitary stalk. 


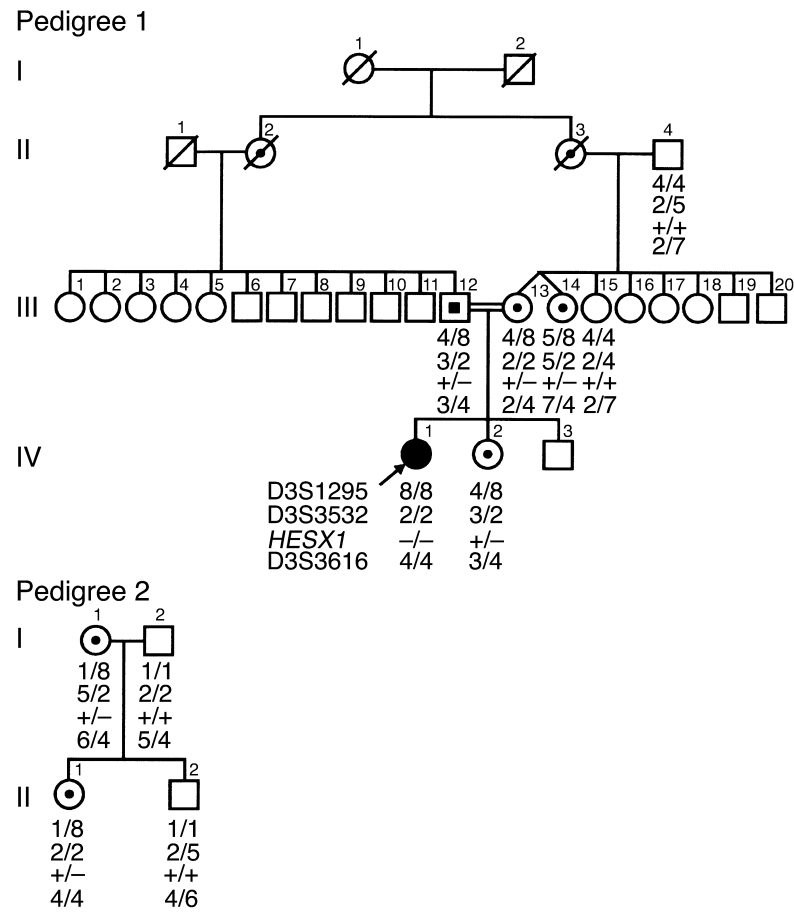

Figure 3

Segregation analysis of the $126 \mathrm{~T}$ mutation within pedigree 1 (the family of the index patient with CPHD) and pedigree 2 (the normal control family) using the highly polymorphic HESX1-flanking markers D3S1295, D3S3532, and D3S3616. The figure shows the presence of haplotype 8, 2, 4 linked to the HESX1(I26T) mutation in individuals from pedigree 1 (III-12, III-13, III-14, IV-1, and IV-2) and pedigree 2 (I-1 and II-1).

the estimated frequency of the 8,2, 4 haplotype was $1.9 \%$ in the normal population. As the interval between the flanking markers of HESX1 is $0.8 \mathrm{cM}$ and the probability of recombination is almost null, we conclude that the haplotype carrying the I26T mutation has been transmitted without recombination from one generation to the next, suggesting that this mutation may have arisen from a common founder.
HESX1(I26T) is associated with impaired transcriptional repression but not DNA binding. Previous studies have shown that HESX1 binds as a dimer with high affinity to the P3 DNA sequence (TAATGAATTA), a synthetic palindrome shown to bind Prd homeodomain proteins $(3,6)$. Electrophoretic mobility shift assays (Figure 4) demonstrate that recombinant HESX1(I26T) binds to DNA bearing a single P3 site. HESX1, HESX1(I26T), and HESX1(50-185), in which the N-terminal 49 AAs of HESX1, including eh1, are deleted, appear to bind with similar DNA-binding affinity at all protein concentrations (Figure 4, and data not shown), whereas HESX1(R160C) does not bind this DNA fragment (Figure 4). The specificity of the DNA binding is shown by competition experiments in which an excess concentration $(15 \mathrm{pM})$ of unlabeled DNA probe competed with radiolabeled probe in the interaction with HESX1 and its mutants (Figure 4, lanes 5, 10, and 12).

While the I26T substitution does not affect DNA binding, Figure 5 shows that this substitution impaired transcriptional repression by HESX1. Cos-7 cells, previously shown to express the corepressor TLE-1 (S. Stifani, unpublished observations; data not shown), were transiently transfected with increasing amounts of DNA encoding HESX1 or HESX1(I26T) fused to the heterologous DNA-binding domain of GAL4. As shown in Figure 5a, these expression vectors were cotransfected along with a reporter containing GAL4 DNA-binding sites upstream of the SV40 promoter activating expression of the luciferase gene. Cotransfection of increasing amounts of GAL4HESX1 produced a dose-dependent suppression of SV40 promoter activity. The I26T mutation results in impaired repression, as expression of increasing amounts of GAL4-HESX1(I26T) shows only modest repression of the SV40 reporter gene. GAL4HESX1(50-185) lacks the amino-terminal HESX1 repression domain, and cotransfection of increasing amounts of plasmid encoding this fusion protein has little effect on reporter-gene transcription. Impaired repression by HESX1(I26T) was not limited to

Table 2

Frequency of alleles for each of the HESX1-linked markers in 30 normal Brazilian individuals

\begin{tabular}{|c|c|c|c|c|c|}
\hline \multicolumn{6}{|c|}{ Allele } \\
\hline \multicolumn{2}{|c|}{ D3S1295 } & \multicolumn{2}{|c|}{ D3S3532 } & \multicolumn{2}{|c|}{ D3S3616 } \\
\hline Fragment size (bp) & Frequency & Fragment size (bp) & Frequency & Fragment size (bp) & Frequency \\
\hline 122 & 0.13 & 234 & 0.083 & 202 & 0.033 \\
\hline 128 & 0.016 & 236 & 0.48 & 204 & 0.150 \\
\hline 130 & 0.016 & 238 & 0.200 & 206 & 0.050 \\
\hline 132 & 0.45 & 240 & 0.033 & 208 & 0.300 \\
\hline 134 & 0.13 & 242 & 0.180 & 210 & 0.160 \\
\hline 136 & 0.05 & 244 & 0.016 & 212 & 0.110 \\
\hline 140 & 0.016 & & & 214 & 0.100 \\
\hline 142 & 0.130 & & & 216 & 0.016 \\
\hline 144 & 0.05 & & & 218 & 0.066 \\
\hline
\end{tabular}

Numbers in boldface indicate the observed frequency of each of the three alleles that give rise to the haplotype 8, 2, 4 associated with the I26T mutation. These figures predict that this haplotype would be present in $1.9 \%$ of the normal population. 


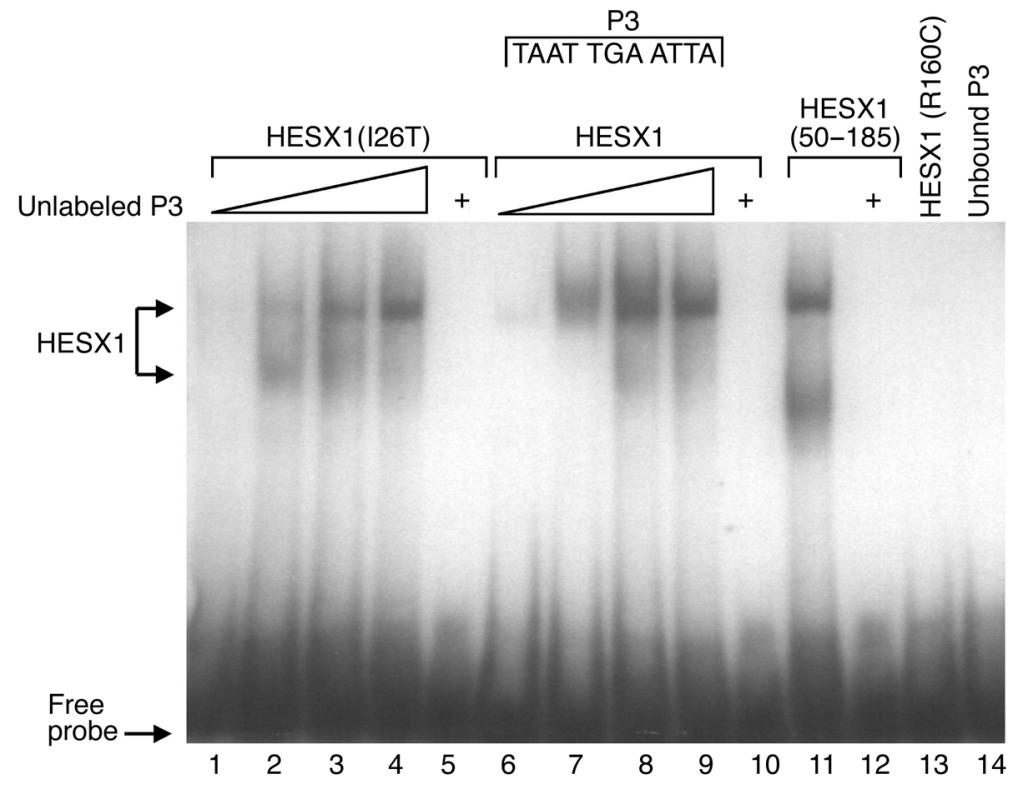

\section{Figure 4}

Electrophoretic mobility shift assay. Increasing amounts of in vitro-translated HESX1(I26T) $(1 \mu \mathrm{l}$, $5 \mu \mathrm{l}, 10 \mu \mathrm{l}$, and $20 \mu \mathrm{l}$ ) were added to the consensus palindromic DNA-binding site P3 (lanes 1-4). Identical amounts of wild-type HESX1 were added to P3 (lanes 6-9). Lane 11 shows addition of the highest amount of HESX1(50-185) (20 $\mu \mathrm{l})$ added to $\mathrm{P} 3$. Lanes 5,10 , and 12 show the highest amounts $(20 \mu \mathrm{l})$ of the respective protein construct [lane 5, HESX1(I26T); lane 10, wild-type HESX1; lane 12, HESX1(50-185)] added to radiolabeled P3 and excess (15 pM) unlabeled P3. Lane 13 represents the addition of $20 \mu \mathrm{l} \mathrm{HESX1(R160C)} \mathrm{to} \mathrm{P3,}$ while lane 14 shows the effect of free probe in the absence of protein. Plus signs at the top of lanes 5 , 10 , and 12 indicate the addition of unlabeled P3.

reporters that contained GAL4-binding sites. Figure $5 \mathrm{~b}$ shows an experiment in which the same expression vectors were cotransfected alongside a reporter containing consensus P3 sites and the homeodomain activator Bix. Activation of this reporter by Bix is efficiently repressed by HESX1, whereas HESX1(I26T) and HESX1(50-185) lead to impaired repression. HESX1(R160C) does not bind DNA but can form heteromeric complexes with homeodomain activators in solution (6). As a result, HESX1(R160C) can act as a dominant negative protein in transient-transfection experiments, in which it forms a complex with a homeodomain activator. In Figure 5b, cotransfection of DNA encoding HESX1(R160C) inhibits the activity of Bix, although not as well as cotransfection of DNA encoding wild-type HESX1. However, HESX1(50-185) (R160C) does not have this activity, and the dominant negative activity of HESX1(I26T)(R160C) is impaired. This is not due to a difference in expression levels, as HESX1, HESX1(I26T), and HESX1(50-185) were all expressed at equivalent levels as determined by Western blot (data not shown). These data implicate the eh1 domain in dimerization and interaction with activator proteins, confirming our previous data (6).

Interaction of HESX1(I26T) with the mammalian Groucho homolog Gro/TLE1 is impaired. As the amino-terminal repression domain contains eh 1 and this region is known to mediate an interaction with the Groucho homolog Gro/TLE, we tested an interaction between HESX1(I26T) and Gro/TLE. The experiments shown in Figure 6 demonstrate that HESX1, but not HESX1(I26T) or HESX1(50-185), can interact with Gro/TLE in vivo. In these experiments, a HESX1 expression vector was cotransfected alongside a $\mathrm{P} 3$ site-containing reporter and a second expression vector encoding a Gro/TLE fusion to modular activation domains derived from the herpes simplex virus transactivator VP16 (Gro/TLE-VP16). As Gro/TLE does not contain a DNA-binding domain, the reporter is only activated when the Gro/TLE-VP16 fusion is recruited to the promoter via an interaction with HESX1. Cotransfection of increasing amounts of the Gro/TLE-VP16 expression vector led to a 14-fold induction of the P3-containing reporter in the presence of HESX1, but not HESX1(50-185). Figure 6 also shows that Gro/TLE-VP16 did not efficiently activate reportergene transcription when cotransfected with HESX1(I26T) (only threefold with the highest concentration of Gro/TLE-VP16). These data were confirmed in coimmunoprecipitation experiments using FLAG-HESX1 or FLAG-HESX1(I26T) transfected into HEK 293A cells expressing endogenous Gro/TLE proteins (Figure 7). The immunoprecipitation of HESX1 with anti-FLAG antibodies (Figure 7b, lane 4 ) resulted in the coimmunoprecipitation of endogenous Gro/TLE (Figure 7d, lane 4). In contrast, immunoprecipitation of HESX1(I26T) did not result in Gro/TLE coimmunoprecipitation, even though HESX1(I26T) was expressed at the same level as HESX1 (Figure 7, b and d, lane 5). The specificity of the interaction of HESX1 and Gro/TLE was shown further by the demonstration that a truncated form of the bHLH protein Hes1, lacking the C-terminal WRPW motif required for Gro/TLE interaction (22), also failed to coimmunoprecipitate with Gro/TLE (Figure 7, b and d, lane 6). These findings demonstrate that the I26T mutation abrogates but does not totally abolish the ability of HESX1 to recruit the Gro/TLE corepressor, and this partial loss of function may account for the relatively mild phenotype associated with this mutation.

\section{Discussion}

We report the first homozygous human mutation within HESX1 in the highly conserved repressor motif eh1, found in a number of developmental repressors, such as Engrailed, Gsc, and Dharma/Bozozok (Dha/Boz), many of which are crucial in anterior patterning. In HESX1, this sequence is contained within an amino-terminal 
repression domain that mediates repression of homeodomain-containing activators. The mutation leads to the substitution of a highly conserved isoleucine residue by threonine within eh1 in HESX1. The phenotype in the patient homozygous for HESX1(I26T) consists of evolving CPHD with a hypoplastic anterior pituitary, a thin pituitary stalk, and an ectopic PPL on MRI scanning. No phenotype was observed in individuals heterozygous for HESX1(I26T), and the homozygous phenotype differs from that previously described for a mutation in the homeodomain [HESX1(R160C)] and the null allele in the mouse (3). Both the mouse mutation and the family carrying HESX1(R160C) exhibit optic nerve hypoplasia and forebrain defects that were present along with early-onset panhypopituitarism. Molecular analysis of HESX1(I26T) suggests that the defect produced by a mutation within eh1 is distinct from the previously characterized HESX1(R160C) mutation in that it impairs transcriptional repression and not DNA binding.

The coregulatory apparatus that mediates Hesx1dependent repression is unknown, although two corepressors have been implicated in repression by Hesx1: nuclear receptor corepressor-1 (N-CoR1) has been linked to repression by the homeodomain $(14,29)$, and the mammalian orthologs of the $D$. melanogaster protein Groucho can stimulate negative regulation via eh1 (13, $15,16,30,31)$. Groucho mediates corepression via association with HDAC and has diverse roles in mediating repression of genes that respond to a number of signaling pathways, including Wnt, Notch, and TGF- $\beta$. Groucho is recruited to a promoter via a protein-protein interaction with sequence-specific DNA-binding domains, some of which contain small peptide motifs WRPW and FS/YIxxIL (eh1) that directly bind Groucho. Eh1 is present in a diverse array of homeodomain proteins and may also be involved in mediating dimerization between homeodomain activators and repressors $(6,17)$.

Previous studies have suggested that eh1 in HESX1 mediates an interaction with other homeodomain-containing proteins to form a complex that can serve as a promoter-specific binding surface for corepressors such as Gro/TLE $(6,13)$. Here, we demonstrate that HESX1, but not HESX1(50-185) or HESX1(I26T), could recruit Gro/TLE. Similarly, HESX1, but not HESX1(50-185), repressed transcription and interacted with the homeodomain activator Bix. HESX1(I26T) was impaired to varying degrees with respect to all of these activities. Taken together, these data support a role for eh1 in mediating heteromeric complex formation between homeodomain proteins and recruitment of Gro/TLE.

The two prd-like homeodomain factors, the Gro/TLE-dependent homeodomain repressor Hesx1, and the transcriptional activator Prop1 exert temporally distinct reciprocal functions during pituitary development (13). Thus, where Hesx 1 expression and Prop1 expression overlap, Hesx 1 may act to suppress Prop1 activity in the developing pituitary. Supporting this hypothesis are data from a transgenic mouse model where Hesx 1 and Gro/TLE1 expression is maintained after $12.5 \mathrm{dpc}$. These mice phenocopy the pituitary defects in Prop1-defective mice, leading to pituitary dysmorphogenesis and loss of the Pou1f1-dependent and gonadotrope lineages (13). Consequently, the phenotype of the patient described in this paper could be
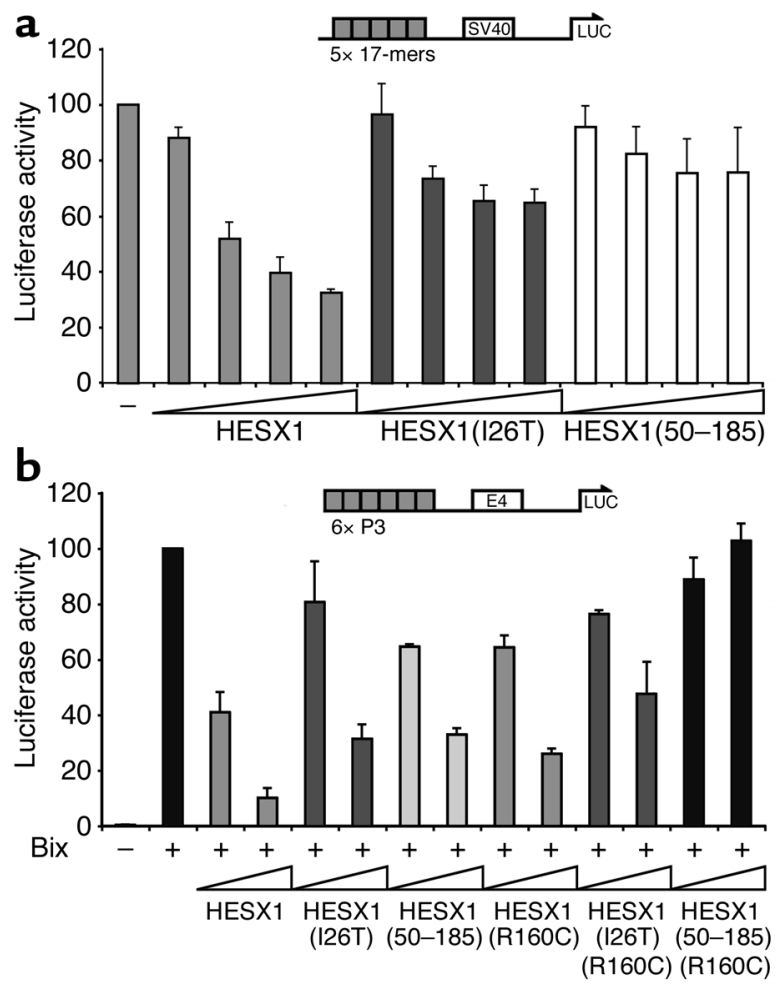

Figure 5

(a) HESX1(I26T) leads to impaired repression at the SV40 promoter. Increasing concentrations of GAL4-HESX1, GAL4-HESX1(I26T), and GAL4-HESX1(50-185) (10 ng, $100 \mathrm{ng}, 250 \mathrm{ng}$, and $500 \mathrm{ng}$ ) were cotransfected with the SV40 promoter reporter construct. GAL4-HESX1 led to a dose-dependent repression of the SV40 promoter $(67.5 \%$ at the highest concentration tested), whereas GAL4HESX1(I26T) was associated with impaired repression (35.2\% at the highest concentration tested) $(P=0.02)$. GAL4-HESX1 (50-185) was associated with $24.4 \%$ repression, which did not achieve statistical significance $(P=0.19)$. The results represent the means of three independent experiments, each performed in triplicate. (b) HESX1(I26T) leads to impaired repression of Bix activation. Two different concentrations (10 $\mathrm{ng}$ and $250 \mathrm{ng}$ ) of GAL4-HESX1, GAL4HESX1(I26T), GAL4-HESX1(50-185), GAL4-HESX1(R160C), GAL4HESX1(I26T)(R160C), and GAL4-HESX1(50-185)(R160C) were cotransfected with a reporter containing six paired-class binding sites upstream of the E4 promoter that drives luciferase expression [(P3)6E4], and with the paired-class activator Bix. GAL4-HESX1 was associated with a tenfold repression of Bix activation at the highest concentration tested, whereas both GAL4-HESX1(I26T) and GAL4HESX1(50-185) led to a threefold repression of Bix activation at the (P3)6E4 promoter. GAL4-HESX1(R160C) was associated with a fourfold repression of Bix activation, whereas the introduction of the I26T mutation [GAL4-HESX1(I26T)(R160C)] or the removal of the N-terminal AAs [GAL4-HESX1(50-185)(R160C)] led to a twofold repression and no repression of Bix activation, respectively. A constant concentration of Bix was used in all experiments (250 $\mathrm{ng}$ ). The results represent the means of three independent experiments, each performed in triplicate. 


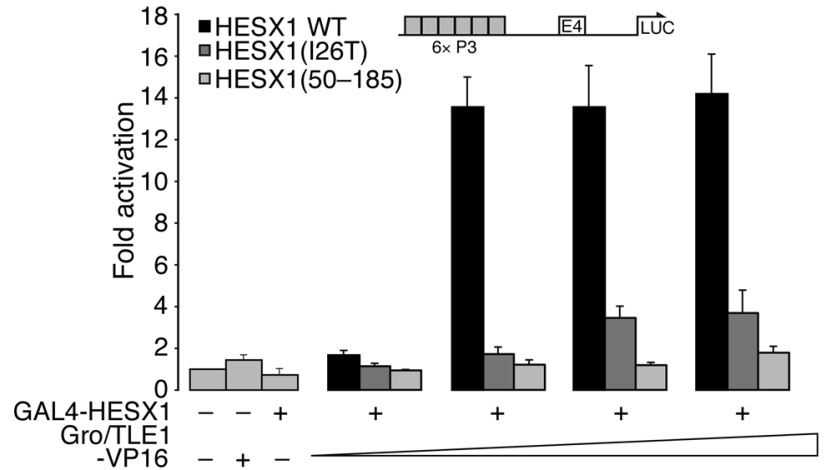

Figure 6

HESX1(I26T) shows impaired interaction with Gro/TLE-VP16 in transient transfection assays. A constant concentration of GAL4-HESX1, GAL4-HESX1(I26T), or GAL4-HESX1(50-185) (250 ng) was cotransfected with increasing concentrations of Gro/TLE-VP16 (10 ng, 100 $\mathrm{ng}, 400 \mathrm{ng}$, and $800 \mathrm{ng}$ ) together with the (P3)E4 reporter construct. GAL4-HESX1 led to a 14-fold activation of (P3)E4 reporter activity when cotransfected with the highest concentration of VP16-Gro/TLE, whereas GAL4-HESX1(I26T) was associated with a threefold activation of the reporter. GAL4-HESX1(50-185) did not lead to any activation of the reporter above base line. Control transfections with each of the expression constructs transfected individually show that, separately, neither GAL4-HESX1 nor Gro/TLE-VP16 can bind and activate the (P3)E4 reporter. The results represent the means of three independent experiments, each performed in triplicate.

explained by the early expression of $P R O P 1$ within the developing pituitary, as observed in transgenic mice in which Prop1 was expressed under the control of the 8-kb Pitx1 regulatory sequences (13). The patient homozygous for HESX1(I26T) presents a milder phenotype than either the patients carrying HESX1(R160C) or the null mouse Hesx 1 allele. The phenotype encompasses evolving hypopituitarism, but with no forebrain defects. This phenotype is similar to that described in association with the heterozygous mutations HESX1(S170L), HESX1(T181A), and HESX1(Q6H). The milder HESX1(S170L) phenotype consisted of IGHD variably associated with an undescended/ectopic posterior pituitary and optic nerve hypoplasia $(5,6)$. HESX1(T181A) was associated with IGHD in association with a nonvisualized posterior pituitary, while HESX1(Q6H) was associated with GH and TSH deficiencies and an undescended/ectopic posterior pituitary (5). The disconnection between the anterior and posterior pituitary, exemplified in our index patient by the ectopic posterior lobe with atrophy of the pituitary stalk, is a hallmark of the majority of human HESX1 mutations as well as the majority of mouse Hes $x 1^{-/-}$ mutations and possibly represents the effects of an early developmental defect within the diencephalon and Rathke's pouch (3). This, to our knowledge, is the first description of evolving TSH and ACTH deficiencies associated with mutations in HESX1. The evolving ACTH deficiency is reminiscent of that observed in some patients with PROP1 mutations (9), and the mechanism, although unknown, might be similar.
The milder clinical phenotype is restricted to the pituitary and displays no defects in forebrain development. However, unlike the null mutation in the mouse, HESX1(I26T) is associated with partial loss of function. HESX1(I26T) can bind DNA and potentially regulate transcription by Gro/TLE-independent mechanisms, possibly accounting for the modest degree of repression observed in Figure 5. Additionally, Figure 6 indicates that the interaction with Gro/TLE is not completely lost, as there is a modest activation of the reporter by HESX1(I26T) cotransfected with Gro/TLE1 fused to VP16.

This contrasts with the properties of HESX1(R160C), which is unable to bind DNA at physiological concentrations and therefore results in loss of function. The resulting phenotype of HESX1(R160C) may thus reflect other regulatory properties of HESX1 lost in the forebrain, independent of eh1. HESX1(I26T) may, on the other hand, also be able to act in conjunction with other repressors in the forebrain to maintain an off state of HESX1 target promoters in the absence of protein-protein interactions that depend on eh1.

Our data also suggest that in the heteromeric repression of homeodomain activators such as Bix, both eh1 and the homeodomain appear to be required for full repression (Figure $5 b$ ). We have previously shown that not only does eh1 mediate repression, but it also

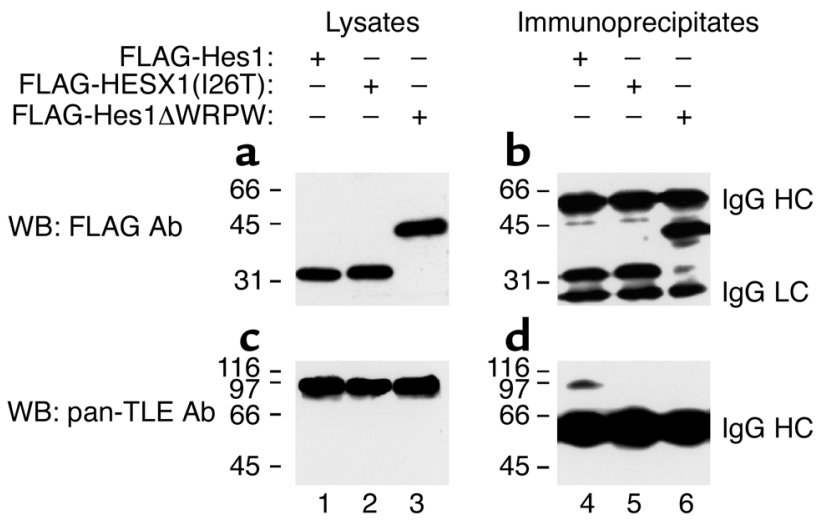

Figure 7

Interaction of HESX1 with Gro/TLE. HEK 293 cells were transfected with plasmids encoding FLAG epitope-tagged HESX1 (lanes 1 and 4), HESX1(126T) (lanes 2 and 5), or a truncated form of the bHLH protein Hes1, lacking the C-terminal WRPW motif (lanes 3 and 6). (b and d) Cell lysates were prepared and subjected to immunoprecipitation with anti-FLAG epitope antibodies, followed by SDSPAGE. ( $\mathbf{a}$ and $\mathbf{c}$ ) One-eighth of each input lysate, collected prior to incubation with antibodies, was also subjected to gel electrophoresis. After transfer to nitrocellulose, Western blotting (WB) was performed with either anti-FLAG ( $\mathbf{a}$ and $\mathbf{b}$ ) or anti-Gro/TLE (pan-TLE; c and d) antibodies. Both HESX1 and HESX1(I26T) migrated as roughly $32-\mathrm{kDa}$ proteins, but only HESX1 coimmunoprecipitated with endogenous Gro/TLE proteins of roughly $95 \mathrm{kDa}$. The specificity of this interaction was demonstrated further by the finding that Hes $1 \triangle$ WRPW also failed to interact with Gro/TLE, as previously shown (22). Positions of migration of the IgG heavy chain (HC) and light chain (LC) are indicated. Positions of size standards are indicated in kilodaltons. 
appears to be critical for dimerization and interaction with homeodomain activators (6).

The present study reveals a further phenotypic category in which HESX1 mutations play a role, namely, evolving CPHD with no midline defects. Comparison of this novel eh1-related phenotype in the pituitary with the more global forebrain defects presented by two children homozygous for a mutation in the homeodomain [HESX1(R160C)] suggests that HESX1 may recruit additional corepressors in the forebrain, but not the pituitary. Additionally, to our knowledge, this is the first report of a mutation within eh 1 in a developmental repressor in humans, with a novel mechanism whereby the interaction with a corepressor, Gro/TLE, is impaired. Studies of the structurefunction relationships of HESX1 mutations can therefore reveal crucial insights into the functional role of the gene in human embryogenesis.

\section{Acknowledgments}

We would like to thank Chin Jia Lin, John Achermann, Meng Li, Gillian Morrison, John S. Parks, Kling Chong, Elena Tzouanacou, and Val Wilson for invaluable advice; Suemi Marui, Ana Elisa C. Billerbeck, and Claudia Costa Leite for significant contributions; and Fernando Kok, Veronica Mericq, Teresa C.A. Vieira, and Mirta Knoepfelmacher for referral of subjects. We would also like to thank Thorsten Gorba, Simona Dariozzi, James Turton, and David MacNay for their assistance. This study was supported by grants from the Fundação de Amparo à Pesquisa do Estado de São Paulo, Brazil (99/08655-7 to L.R. Carvalho and 00/14092-4 to I.J.P. Arnhold) and the Conselho Nacional de Desenvolvimento Científico e Tecnológico, São Paulo, Brazil (301246/95-5 to B.B. Mendonca and 300859/98-8 to I.J.P. Arnhold); the Wellcome Trust (to J.M. Brickman); the Canadian Institute of Health Research (grant to S. Stifani), Quebec, Canada; and the Medical Research Council (MRC; Career Establishment Grant to M.T. Dattani), United Kingdom. S. Stifani is a scholar of the Fonds de la Recherche en Santé du Quebec. J.M. Brickman is a Wellcome Trust Research Career Development Fellow.

1. Schwind, J.L. 1928. The development of the hypophysis cerebri of the albino rat. Am. J. Anat. 41:295-315.

2. Kaufman, M.H. 1992. An atlas of mouse development. Academic Press, Elsevier Science. Oxford, United Kingdom. 39-210.

3. Dattani, M.T., et al. 1998. Mutations in the homeobox gene HESX1/Hesx1 associated with septo-optic dysplasia in human and mouse. Nat. Genet. 19:125-133.

4. Martinez-Barbera, J.P., Rodriguez, T.A., and Beddington, R.S. 2000. The homeobox gene Hesx 1 is required in the anterior neural ectoderm for normal forebrain formation. Dev. Biol. 223:422-430.

5. Thomas, P.Q., et al. 2001. Heterozygous HESX1 mutations associated with isolated congenital pituitary hypoplasia and septo-optic dysplasia. Hum. Mol. Genet. 10:39-45.

6. Brickman, J.M., et al. 2001. Molecular effects of novel mutations in Hesx1/HESX1 associated with human pituitary disorders. Development. 128:5189-5199.

7. Tajima, T., et al. 2003. Sporadic heterozygous frameshift mutation of HESX1 causing pituitary and optic nerve hypoplasia and combined pituitary hormone deficiency in a Japanese patient. J. Clin. Endocrinol. Metab. 88:45-50.

8. Wu, W., et al. 1998. Mutations in PROP1 cause familial combined pituitary hormone deficiency. Nat. Genet. 18:147-149.

9. Mendonca, B.B., et al. 1999. Longitudinal hormonal and pituitary imaging changes in two females with combined pituitary hormone deficiency due to deletion of A301,G302 in the PROP1 gene. J. Clin. Endocrinol. Metab. 84:942-945.

10. Gage, P.J., et al. 1996. The Ames dwarf gene, df, is required early in pituitary ontogeny for the extinction of Rpx transcription and initiation of lineage-specific cell proliferation. Mol. Endocrinol. 10:1570-1581.

11. Hermesz, E., Mackem, S., and Mahon, K.A. 1996. Rpx: a novel anteriorrestricted homeobox gene progressively activated in the prechordal plate, anterior neural plate and Rathke's pouch of the mouse embryo. Development. 122:41-52.

12. Sornson, M.W., et al. 1996. Pituitary lineage determination by the Prophet of Pit-1 homeodomain factor defective in Ames dwarfism. Nature. 384:327-333.

13. Dasen, J.S., et al. 2001. Temporal regulation of a paired-like homeodomain repressor/TLE corepressor complex and a related activator is required for pituitary organogenesis. Genes Dev. 15:3193-3207.

14. Xu, L., et al. 1998. Signal-specific co-activator domain requirements for Pit-1 activation. Nature. 395:301-306.

15. Jimenez, G., Paroush, Z., and Ish-Horowicz, D. 1997. Groucho acts as a corepressor for a subset of negative regulators, including Hairy and Engrailed. Genes Dev. 11:3072-3082.

16. Jimenez, G., Verrijzer, C.P., and Ish-Horowicz, D. 1999. A conserved motif in goosecoid mediates groucho-dependent repression in Drosophila embryos. Mol. Cell. Biol. 19:2080-2087.

17. Mailhos, C., et al. 1998. Drosophila Goosecoid requires a conserved heptapeptide for repression of paired-class homeoprotein activators. Development. 125:937-947.

18. Osorio, M.G., et al. 2002. Pituitary magnetic resonance imaging and function in patients with growth hormone deficiency with and without mutations in GHRH-R, GH-1, or PROP-1 genes. J. Clin. Endocrinol. Metab. 87:5076-5084.

19. Brickman, J.M., Adam, M., and Ptashne, M. 1999. Interactions between an HMG-1 protein and members of the Rel family. Proc. Natl. Acad. Sci. U. S. A. 96:10679-10683.

20. Brickman, J.M., Jones, C.M., Clements, M., Smith, J.C., and Beddington, R.S. 2000. Hex is a transcriptional repressor that contributes to anterior identity and suppresses Spemann organiser function. Development. 127:2303-2315.

21. Wang, J.C., et al. 2000. Transducin-like enhancer of split proteins, the human homologs of Drosophila groucho, interact with hepatic nuclear factor 3beta. J. Biol. Chem. 275:18418-18423.

22. McLarren, K.W., Theriault, F.M., and Stifani, S. 2001. Association with the nuclear matrix and interaction with Groucho and RUNX proteins regulate the transcription repression activity of the basic helix loop helix factor Hes1. J. Biol. Chem. 276:1578-1584.

23. Nuthall, H.N., Husain, J., McLarren, K.W., and Stifani, S. 2002. Role for Hes1-induced phosphorylation in Groucho-mediated transcriptional repression. Mol. Cell. Biol. 22:389-399.

24. Stifani, S., Blaumueller, C.M., Redhead, N.J., Hill, R.E., and ArtavanisTsakonas, S. 1992. Human homologs of a Drosophila Enhancer of split gene product define a novel family of nuclear proteins. Nat. Genet. 2:119-127.

25. Gruneiro-Papendieck, L., Chiesa, A., Martinez, A., Heinrich, J.J., and Bergada, C. 1998. Nocturnal TSH surge and TRH test response in the evaluation of thyroid axis in hypothalamic pituitary disorders in childhood. Horm. Res. 50:252-257.

26. Mehta, A., et al. 2003. Central hypothyroidism: variable responses to the TRH test. Poster no. P3-537 presented at: 85th Annual Meeting of the American Endocrine Society. June 19-22. Philadelphia, Pennsylvania, USA.

27. Simmons, G.E., Suchnicki, J.E., Rak, K.M., and Damiano, T.R. 1992. MR imaging of the pituitary stalk: size, shape, and enhancement pattern. AJR Am. J. Roentgenol. 159:375-377.

28. Argyropoulou, M., Perignon, F., Brauner, R., and Brunelle, F. 1992. Magnetic resonance imaging in the diagnosis of growth hormone deficiency. J. Pediatr. 120:886-891.

29. Laherty, C.D., et al. 1998. SAP30, a component of the mSin3 corepressor complex involved in $\mathrm{N}$-CoR-mediated repression by specific transcription factors. Mol. Cell. 2:33-42.

30. Chen, G., and Courey, A.J. 2000. Groucho/TLE family proteins and transcriptional repression. Gene. 249:1-16.

31. Fisher, A.L., and Caudy, M. 1998. Groucho proteins: transcriptional corepressors for specific subsets of DNA-binding transcription factors in vertebrates and invertebrates. Genes Dev. 12:1931-1940. 